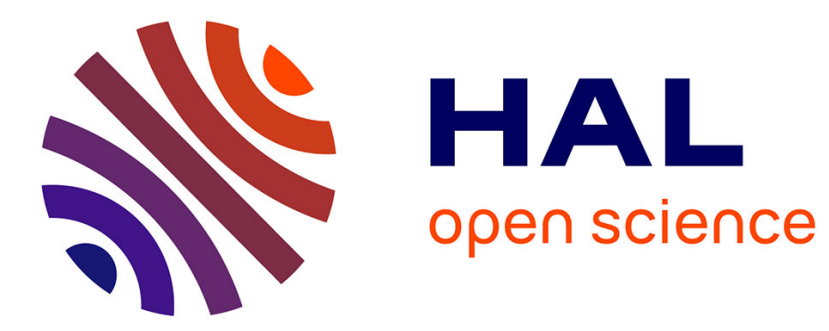

\title{
Full-field measurements with Digital Image Correlation for vibro-impact characterisation
}

Robin Chabrier, Emeline Sadoulet, Gael Chevallier, Emmanuel Foltête, Thomas Jeannin

\section{- To cite this version:}

Robin Chabrier, Emeline Sadoulet, Gael Chevallier, Emmanuel Foltête, Thomas Jeannin. Full-field measurements with Digital Image Correlation for vibro-impact characterisation. Mechanical Systems and Signal Processing, 2021, 156, pp.107658 (16). hal-03359915

\section{HAL Id: hal-03359915 https://hal.science/hal-03359915}

Submitted on 30 Sep 2021

HAL is a multi-disciplinary open access archive for the deposit and dissemination of scientific research documents, whether they are published or not. The documents may come from teaching and research institutions in France or abroad, or from public or private research centers.
L'archive ouverte pluridisciplinaire HAL, est destinée au dépôt et à la diffusion de documents scientifiques de niveau recherche, publiés ou non, émanant des établissements d'enseignement et de recherche français ou étrangers, des laboratoires publics ou privés. 


\title{
Full-field measurements with Digital Image Correlation for vibro-impact characterisation
}

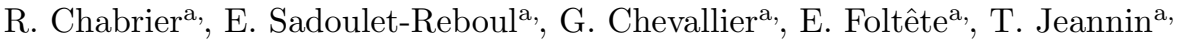 \\ ${ }^{a}$ Univ. Bourgogne Franche-Comté, FEMTO-ST Institute, CNRS/UFC/ENSMM/UTBM, Department of Applied Mechanics, 24 \\ chemin de l'Epitaphe, 25000 Besancon, France
}

\begin{abstract}
Vibro-impact (VI) based control strategies exploit non-linear phenomena occurring during impacts between oscillating masses and vibrating structures to mitigate vibrations. They are known to be effective on a wide frequency range, in harsh environnement, and they are eco-friendly in comparison to some visco-elastic polymers used for the same application. Different assumptions emerge to explain energy dissipation such as material and contact effects, or dynamic energy transfers between the structure and the oscillating masses acting as non-linear absorbers. As classical vibratory measurement tools do not allow to accurately and easily measure what happens in the contact area and the motion of the absorber, it is difficult to validate and quantify the phenomenon. The purpose of the study is to take advantage of full field measurements of the vibro-impact phenomenon to characterise a vibro-impact absorber and to better understand the interactions occurring during impact. To reach this objective, a dedicated experimental set-up has been designed and a method involving Digital Image Correlation (DIC) and a high-speed camera is used to capture and reconstruct the motion of the oscillating mass. The characterisation allows to estimate the Coefficient Of Restitution (COR) and friction forces, and provides data that are used in two different contact modelling strategies based on the COR and on the Hertz contact theory. The quantities identified in this paper enable to better understand the physical phenomena at stakes in a VI absorber.

Keywords: Vibro-impact, Digital Image Correlation, Coefficient of Restitution, Hertz-contact, high-speed camera, full-field measurement.
\end{abstract}

\section{Introduction}

VI absorbers consist of free oscillating masses integrated in a main structure to reduce its vibrations. It is known to be effective on a wide frequency range and in harsh environments. Their effectiveness in mitigating the vibrations relies on the interaction between each oscillating mass and the main structure that occurs during the

5 impacts. Mostly, two assumptions emerge to explain energy dissipation : contact effects such as plastic deformation during the repeated impacts [1] or viscous damping during the contact phase [2] on the one hand, and dynamic effects leading to a high frequency energy transfer and to a faster vibration mitigation [3] on the other hand. In the last case, the vibrational energy of the main structure is redistributed within the modal space from low to high modes that possess higher energy dissipation efficiency.

Email address: robin.chabrier@univ-fcomte.fr (R. Chabrier) 
To better understand the physics of VI phenomenon, it is interesting to adopt an experimental approach. However, several difficulties must be overcome. Impact phenomenon is non-linear, and occurs on a very short time scale what makes its study particularly challenging. Moreover, VI absorbers are often difficult to equip with measuring instruments, especially for the free oscillating masses. Therefore, non-contact techniques could offer an interesting alternative. Among these techniques, DIC has many interests for VI study to measure the displacements that cannot be measured with an accelerometer nor a laser vibrometer. Indeed, it is a low noise method (accuracy is below pixel size) and combined with a high-speed camera, it becomes possible to capture the dynamic behaviour of a VI system. In solid mechanics, DIC and full-field measurement techniques have paved the way to new approaches to measure displacement, velocity or strain field and have a growing interest thanks to the advent of high-speed cameras that allow to get pictures even for dynamic system with short typical variation time. Many applications are found for dynamic testing in combination with high-speed or ultra-high-speed cameras [4. This enables the visualisation of transient deformation [5, the development of local deformation [6, 7, or phase transformation during dynamic test on shape memory alloys [8]. Moreover, measuring 3-D displacement field becomes possible if two cameras are used [4].

The purpose of this paper is to take advantage of these methods to reconstruct the motions of both the main structure and the oscillating mass, and to compare different contact modelling strategies.

Full-field measurement techniques have already been set up in the context of granular materials to measure the coefficient of restitution of impact between particles and between wall and particles, combined with different methods. In the following articles, authors have built a special apparatus to film the collision between particles or between a particle and a wall with a high-speed camera. In [9], 10, and [1], the authors can calculate the COR from the height of the rebounds or free flights duration of the particle. These quantities are directly derived from the images obtained from the high speed cameras. In [12, the COR is calculated with the averaged velocities obtained by the displacement over the duration between successive images. Other authors like [13] and [14] have used an energetic definition of the COR and need both translational and rotational velocities of the particle. In the first case, the authors have used the Image Pro Plus software to track the particle and calculate the velocities. In the second case, the locations and velocities of the particle are determined using a particle tracking velocimetry method. In all these cases, calculations are made to obtain the velocities necessary to obtain the COR : they are obtained from the measure of the height of rebound, from the duration between impacts, or from the simple ratio of displacement and duration between images. But the entire motion of the particle is not considered, what can result in a lack of accuracy if only considering the experimental data just before and after impact. The total motion of the particle provides accurate information to estimate displacements and velocities, especially in the case of noisy measurements. This statement is the basis of the method that will be presented in this work.

In the field of vibro-impact, full-field measurement with a high speed camera has been used in [15] to visualise VI regimes between gears. According to the authors, the video post-processing allows measurement of the instantaneous position of the gears from the shape recognition. Recently, a simple method using limited experimental information 45 to reconstruct the motions of the oscillating mass of a VI absorber and measure the COR has been presented in [16]. The authors used a laser sensor and an accelerometer to measure the position of the main structure and its acceleration respectively. From this, the velocity of the main structure can be derived, and the impact time is 
given by the discontinuities on the acceleration signal. Knowing the position of the oscillating mass at impact, its motion can be reconstructed assuming a friction coefficient between the oscillating mass and the main structure. Nevertheless, the motion of the oscillating mass is not directly measured as no visualisation of the ball is possible. Thus no information about the friction coefficient can be provided from this experimental set-up. Moreover, the uncertainty on the friction coefficient leads to uncertainty on the velocity estimation, necessary to calculate the COR. At last, the authors noticed a different coefficient of restitution on the left and right sides of their VI absorber prototype, which is an interesting but unexplained phenomenon. The same observation is reported here.

In this paper, a dedicated set-up has been designed composed of a ball oscillating in a cavity, reproducing the behaviour of a vibro-impact absorber. A method is proposed to reconstruct the motion of the oscillating mass and the main structure using DIC with a single high-speed camera. This method is used to reach the following objectives :

- evaluate the performance of DIC for experimental VI characterisation ;

- use measurements as input in two modelling strategies based on the coefficient of restitution and on a Hertzian contact.

The tracking of the displacement of the main structure and the oscillating mass is realised. Models are assumed for the displacement of both the ball and the structure, and their parameters are identified from curve fitting. The advantage of this is that the velocities can be easily and accurately obtained by analytical derivation without amplifying the noise on the measures. Then, these results allow to characterise the VI absorber and are used as inputs to run simulations of the COR model and Hertz damped contact model.

The paper is organised as follows. Section 2 describes the experimental set-up designed for this study, presents the DIC technique and the post-processing of measurements. Section 3 presents the modelling of the VI problem, and the identification of some of its parameters. Section 4 presents the numerical results. In section 5, uncertainties on the COR are discussed.

\section{DIC for VI measurement}

\subsection{Design of a set-up and instrumentation}

For this study, an experimental set-up consisting of a ball enclosed between two plates has been designed (Fig. 1).

The main structure is made of two plates separated by an adjustable distance and maintained by three holding rods. The structure is rigid enough to ensure that the first natural frequencies cannot be excited in the frequency range of the tests $[22 \mathrm{~Hz}-30 \mathrm{~Hz}]$. For this purpose, the set-up was designed using numerical modal analysis. The ball is guided to ensure vertical movement by three guiding rods, so that it is possible to visualise the entire motion of the ball during its oscillation. The main structure has a sine wave motion imposed by a Dataphysics shaker (1000

$\mathrm{N}$ maximum sine force peak). An adapter plate has also been used to fasten the set-up on the shaker.

Two accelerometers are stuck on each plate to monitor the acceleration of the plates and analyse the impact signals. A high speed camera with a $1000 \mathrm{fps}$ frame rate is used during the test to visualise the oscillation of the 


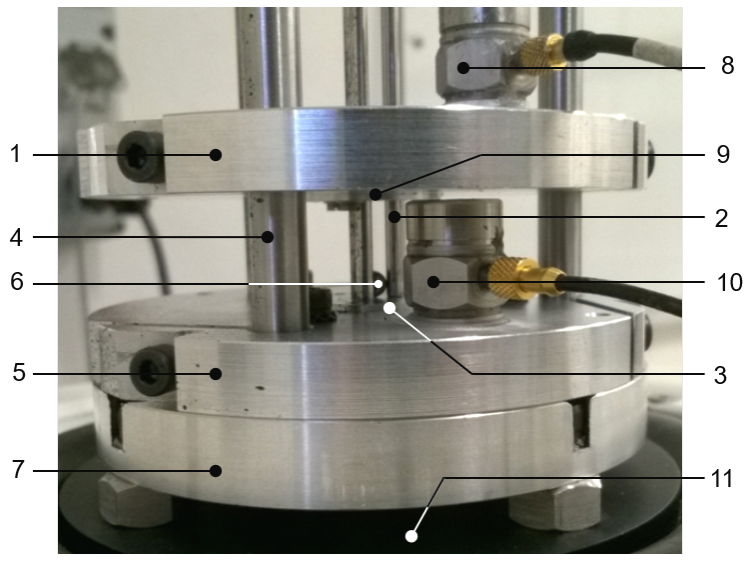

(a) Photo of the experimental set-up

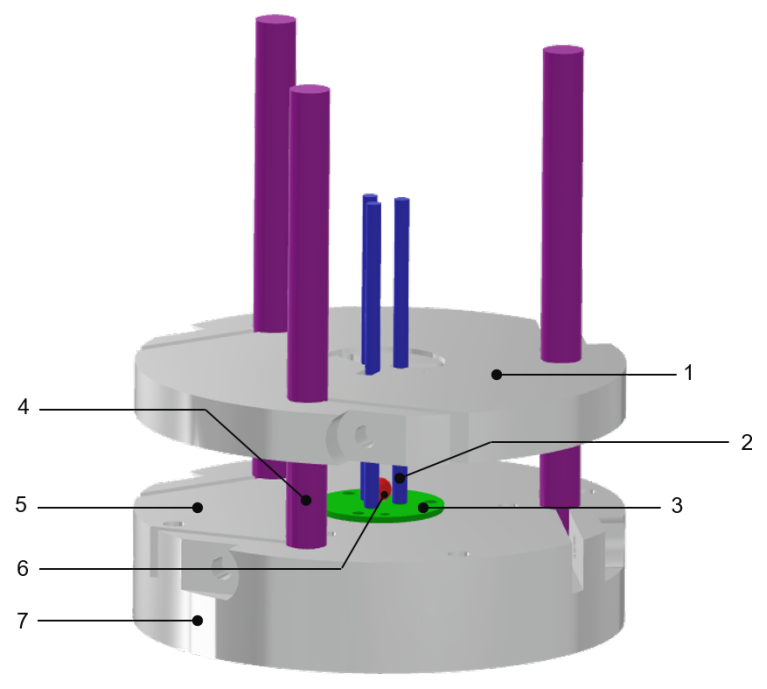

(b) CAD view

Figure 1: Experimental set-up. 1. upper plate ; 2. guiding rod ; 3. lower disk shaped part : 4. holding rod ; 5. lower plate ; 6 . ball ; 7 . adapter plate ; 8. upper accelerometer ; 9. upper disk shaped part ; 10. lower accelerometer ; 11. shaker

ball between the two impact plates. Some photos extracted from the videos are represented in Fig. 2, where it is possible to see the ball going up, colliding the upper plate and going down.

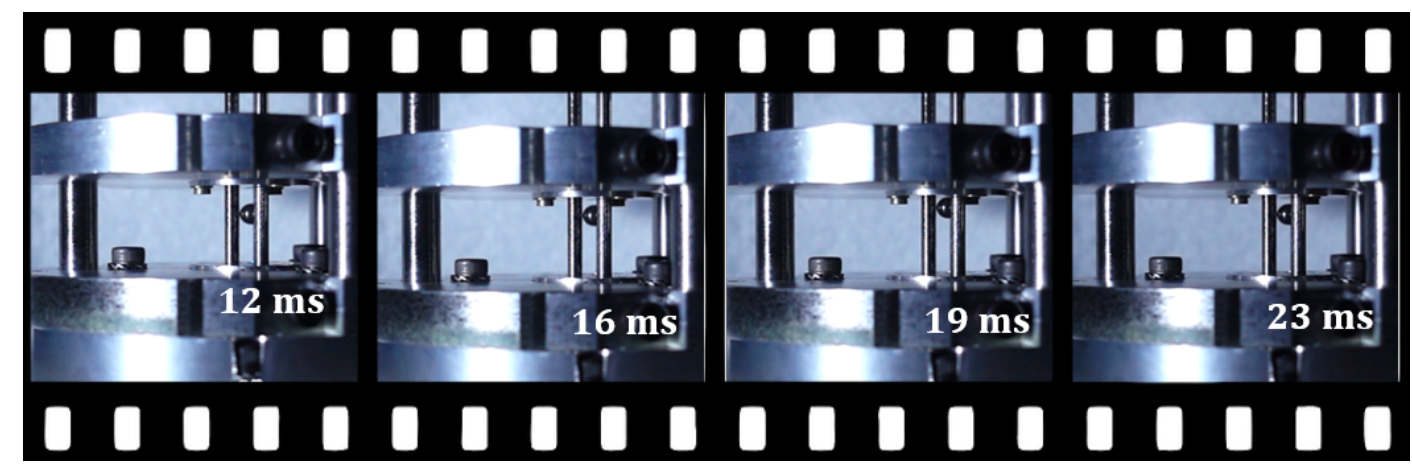

Figure 2: Images extracted from the video recorded with the high-speed camera

\subsection{Description of the experiments}

Different parameters were varied during the experiments, namely the frequency of the excitation and the acceleration (which is directly related to the magnitude of displacement of the shaker). These different parameters are summarised in Table 1. Each test was run once and successively. One test lasts approximately 1,5 seconds, producing 1500 frames to analyse. The frequency and the acceleration of the shaker were chosen in order to obtain VI regimes with two impacts per cycle, one on the upper plate and one on the lower plate.

\subsection{Digital Image Correlation and motion reconstruction}

In order to reconstruct the motion of the main structure and the motion of the oscillating ball, a Digital Image Correlation (DIC) technique is used to follow the motions of both the ball and the structure. Two zones containing 


\begin{tabular}{|c|c|c|}
\hline Test number & $f_{e x c}$ & Max displacement \\
\hline Test $\mathbf{1}$ & $22 \mathrm{~Hz}$ & $27 \mathrm{~mm}$ \\
\hline Test $\mathbf{2}$ & $22 \mathrm{~Hz}$ & $35 \mathrm{~mm}$ \\
\hline Test $\mathbf{3}$ & $22 \mathrm{~Hz}$ & $21 \mathrm{~mm}$ \\
\hline Test $\mathbf{4}$ & $30 \mathrm{~Hz}$ & $27 \mathrm{~mm}$ \\
\hline
\end{tabular}

Table 1: Parameters for the tests

a mesh of points are defined corresponding to the ball and to the main structure (see Fig. 3). A 30 pixels wide subset centred on each point of the mesh is defined. After the displacement, the subset is localised in the new picture thanks to a cross-correlation calculation. The principle of the cross-correlation to determine the displacement of a subset in a image is as follows. Let's consider two subsets : the reference subset, or subset 1, and the current subset, or subset 2 . In the present case, each subset contains 30x30 pixels and each pixel is represented by a gray level intensity. Let's call $I_{1}(x, y)$ the function that gives the gray level intensity of the pixel of coordinate $(x, y)$ belonging to the reference subset ( $x$ and $y$ represent a number of pixels), and $I_{2}(x, y)$ the same function for the current subset. The Zero-mean Normalised Cross Correlation function used in Matlab is defined as [17] :

$$
\gamma(x, y)=\frac{\sum_{i, j}\left[I_{1}(x+i, y+j)-\bar{I}_{1}(x, y)\right]\left[I_{2}\left(x+d_{x}+i, y+d_{y}+j\right)-\bar{I}_{2}(x, y)\right]}{\sqrt{\sum_{i, j}\left[I_{1}(x+i, y+j)-\bar{I}_{1}(x, y)\right]^{2}} \sqrt{\sum_{i, j}\left[I_{2}\left(x+d_{x}+i, y+d_{y}+j\right)-\bar{I}_{2}(x, y)\right]^{2}}}
$$

where $i, j$ scan all the pixels of the subset, $\bar{I}_{1}(x, y)$ and $\bar{I}_{2}(x, y)$ are the average intensity of the pixels in the considered subset ( 1 or 2$), d_{x}$ and $d_{y}$ represent the displacements to determine : they must correspond to the maximum of the cross-correlation function. This criterion is nonsensitive to intensity variations between the reference and the current subset, as it is normalised with averaged intensity. Therefore, the displacements of each point of the mesh is obtained (see Fig. 4). In the present case, as no deformation is expected, the displacement of the ball and of the main structure are calculated as the average displacement of the points of each mesh. Knowing the length of the cavity, it is possible to deduce the displacement of the upper and lower plate, and to position them relatively to the ball displacement. Such results are presented in Fig. 5 .

\subsection{Signals from accelerometers : post-processing}

Two accelerometers were stuck on each plate, (see Fig. 1a) during the dynamic tests. They were used to monitor the acceleration of the main structure on the one hand, and to study the post-impact vibrations in the frequency domain on the other hand.

Typical acceleration signals recorded by the accelerometers are shown in Fig. 6a. The sampling frequency is $50 \mathrm{kHz}$. One can clearly distinguish the low frequency sine wave acceleration corresponding to the excitation of the shaker, and the repeating pattern of high frequency vibrations due to the impacts of the ball on the structure. In order to analyse the frequency content of the impact signals, it is necessary to focus especially on the high frequency content of the acceleration signals. Therefore, the following method is applied. To begin with, signals were filtered using a high-pass filter with a $100 \mathrm{~Hz}$ cut-off frequency to remove the low frequency excitation (see

Fig. 6). The frequency of the excitation was no more than $30 \mathrm{~Hz}$. Next, each transient vibration due to impact is 


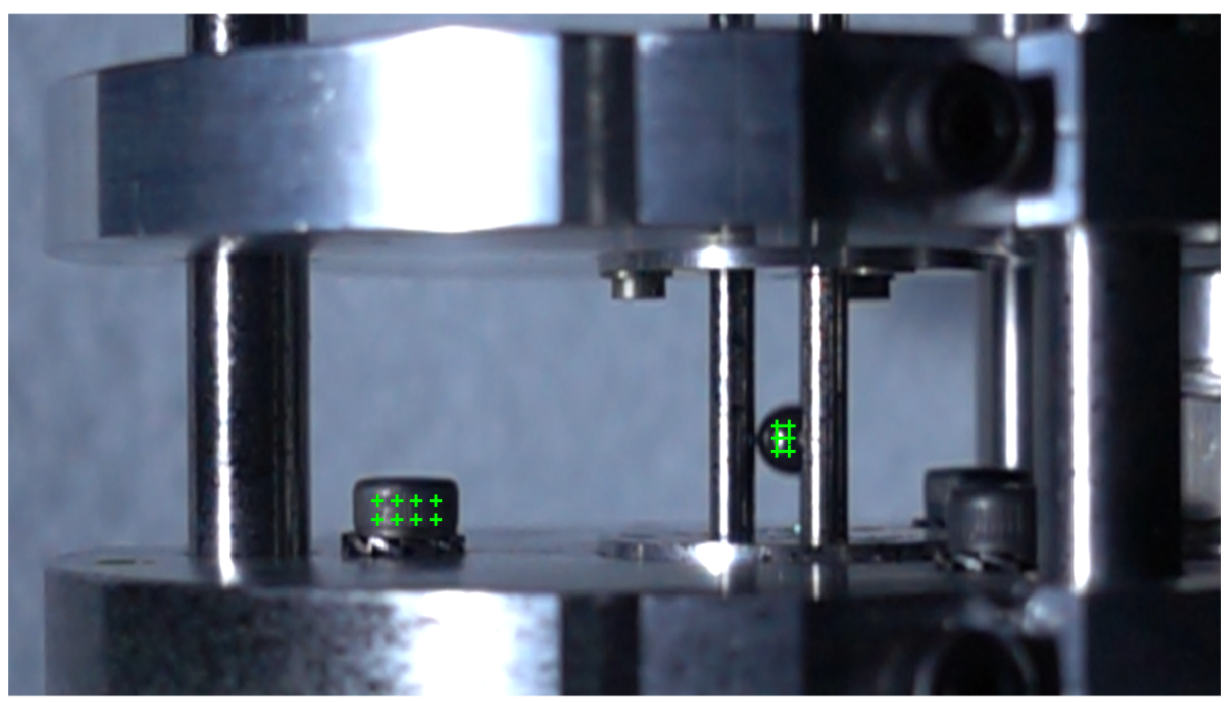

Figure 3: Mesh of points used for the DIC

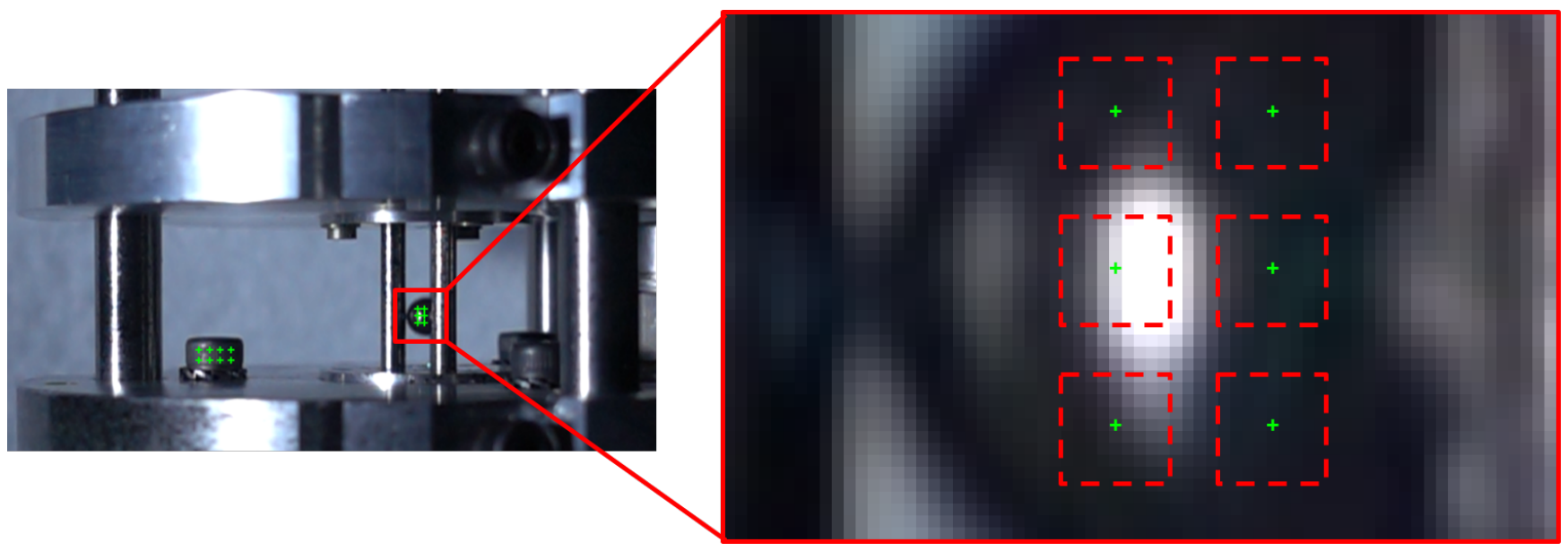

(a) Meshes for DIC : general view

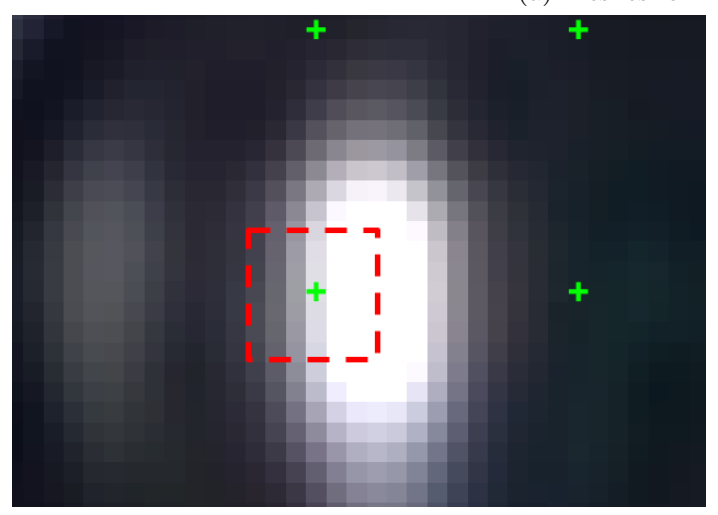

(b) Reference configuration

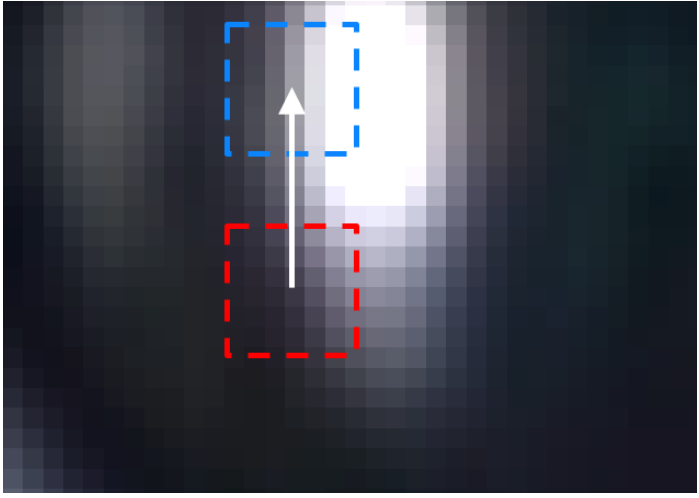

(c) Configuration after displacement

Figure 4: Principle of DIC. (a) and (b) red squares : subsets. (c) Red square : reference subset. Blue square : matched subset after displacement. White arrow : displacement vector 


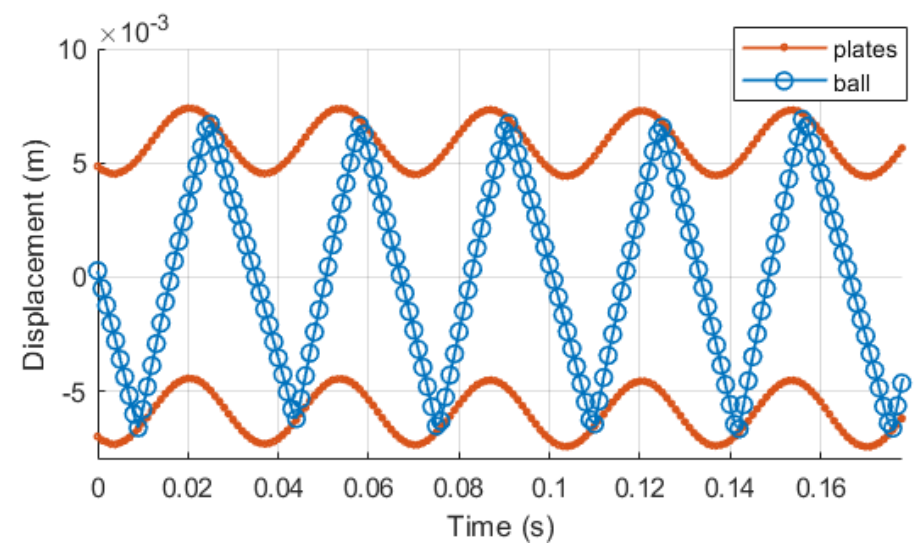

Figure 5: Experimental displacements obtained from DIC

isolated considering a signal length equal to a half-period of the excitation signal. The Fourier transform of each impact was calculated using the fft function in Matlab, and the results in the frequency domain were averaged using all the impacts recorded. The frequencies visible in the Fourier spectrum in Fig. 7 should correspond to the natural frequencies of the structure. This can be explained considering energy transfer between the ball and the main structure. In [3, the authors have shown numerically that when using a VI absorber at a beam end excited on its first mode, the activation of the VI absorber leads to excitation of higher vibrational modes of the beam. This non-linear behaviour can be interpreted as a high frequency energy transfer : the energy initially localised on the first mode of the beam is transferred to higher modes because of impacts. A similar phenomenon is probably observed here, even if it was not confirmed by further analyses : an experimental modal analysis was difficult to perform because of the impact location that is impossible to reach with a classical hammer, and modes identification using a numerical modal analysis turned out to be difficult because several modes are located in a same frequency range. Nevertheless, the Fourier transform obtained in Fig. 7 confirms the non-linear behaviour of the vibro-impact phenomenon.

\section{Vibro-impact modelling and parameter identification}

\subsection{Modelling and parametrisation}

A scheme of the experimental set-up studied in the previous section is represented in Fig. 8, In [18, the authors have analysed the influence of gravity on stability conditions for the same device than the one studied here. Even if stability conditions are out of the scope of this paper, the equations of motions are the same than in the present case.

$Y_{s}(t)$ and $Y_{b}(t)$ denote the position of the main structure (i.e the cavity) and the ball, respectively. The total distance travelled by the ball in the cavity is 2.b. As the signal excitation of the shaker is a sine wave motion, the displacement of the main structure is supposed to be given by Eq. 21:

$$
Y_{s}(t)=A \cdot \sin (\omega \cdot t+\varphi)+B
$$

where $A$ is the amplitude, $\omega$ is the pulsation, $\varphi$ is the phase and $B$ is the offset, what seems to be in accordance with the experimental displacements of the plates shown in Fig. 5 


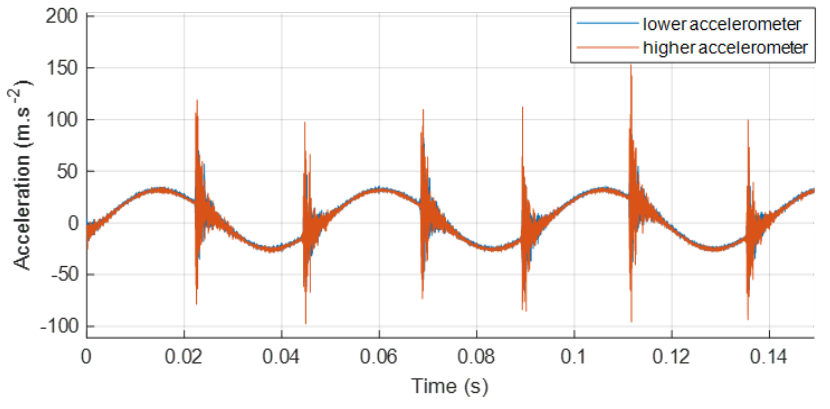

(a) Acceleration signals before filtering

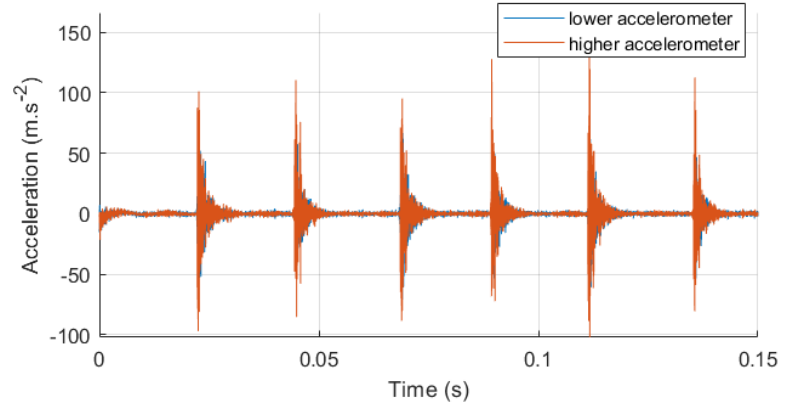

(b) Acceleration signals filtered.

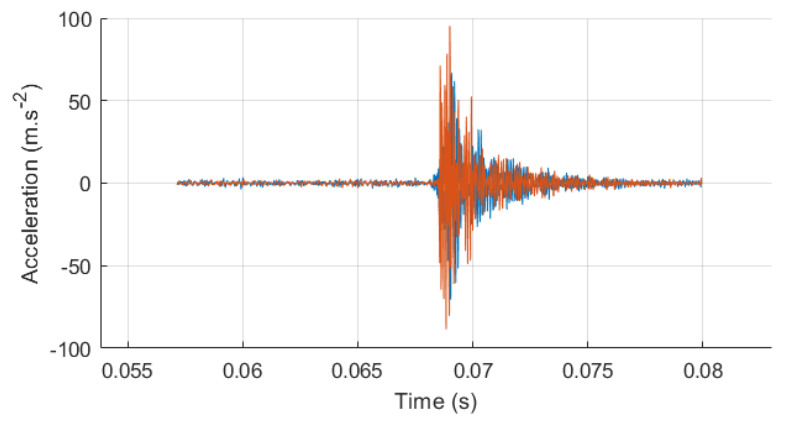

(c) One impact signal isolated.

Figure 6: Post-processing of acceleration signals

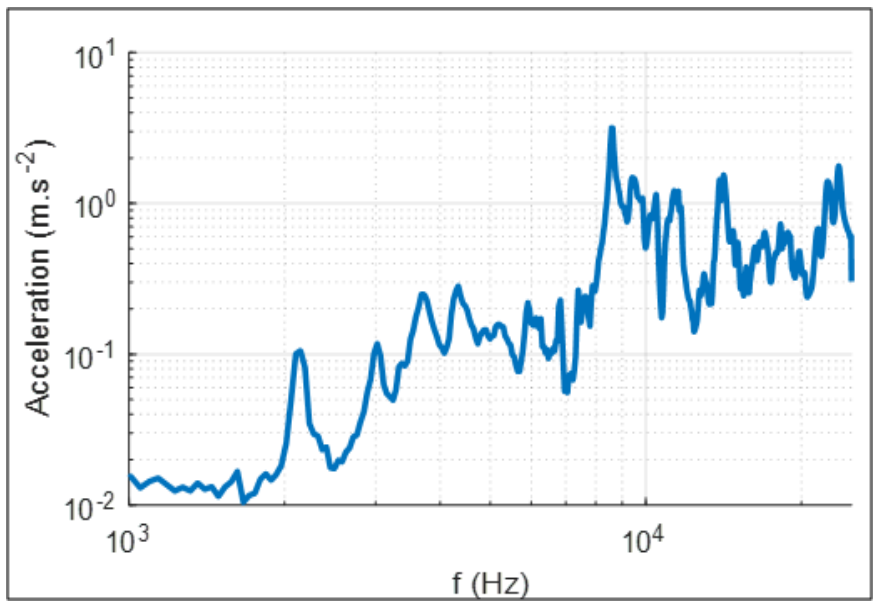

Figure 7: Fourier transform of impacts signals 


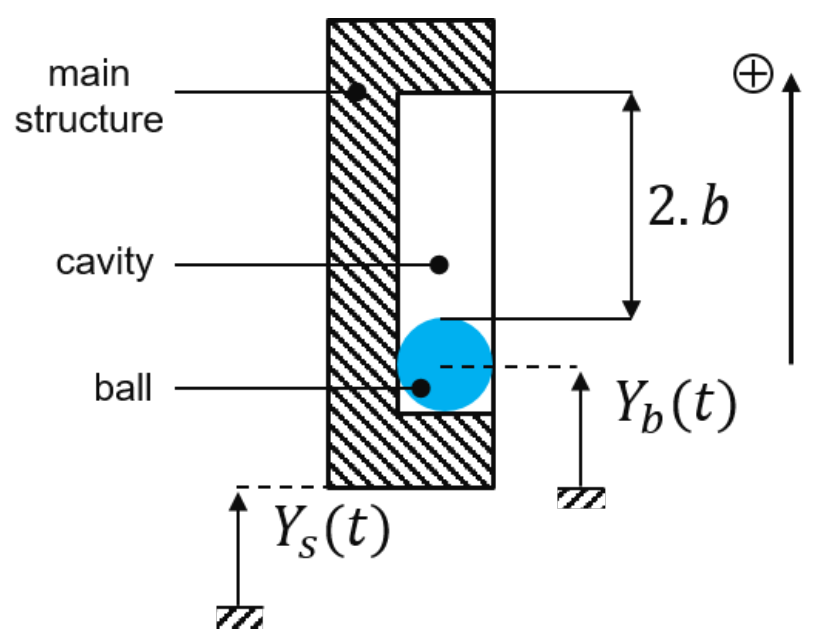

Figure 8: Schema of the VI absorber

The motion of the ball can be separated into two phases : the free flight phase and the contact phase. Looking closely to Fig. 5, the displacement of the ball is not perfectly linear during the free flight phases. It makes sense as the ball is submitted to gravity because of its vertical motion. Therefore, its displacement is assumed to be quadratic with respect to time and given by Eq. 3

$$
Y_{b}^{i}=a_{i} \cdot t^{2}+b_{i} \cdot t+c_{i} \quad t \in\left[t_{i} ; t_{i+1}\right]
$$

for the i-th free flight phase.

With such a model, constant forces acting on a ball are represented such as weight and friction, and the equation of motion governing the displacement of the ball is :

$$
m_{b} . \ddot{y}=-m_{b} . g+F_{\text {friction }}
$$

where $m_{b}$ is the mass of the ball, $g$ is the acceleration of gravity, and $F_{\text {friction }}$ represents the friction force that may apply on the ball when sliding in the cavity.

\subsection{Contact modelling strategies}

Two contact models are used here : the first one is based on the COR, and the second one is a Hertz-damped contact model. In the first approach, the contact phase is assumed infinitely short, and the physical phenomena that occur during impact are wrapped in a coefficient of restitution $e$ defined in Eq. 5 that traduces the energy loss during the impact : $e=1$ represents perfectly elastic impacts (no energy is dissipated during the impact), while $e=0$ represents perfectly inelastic impacts (there is no restitution of energy after the impact, the solids remain in contact). Therefore, each time an impact occurs, the relative speed before and after impact are related by the following equation :

$$
\dot{Y}_{b}^{+}-\dot{Y}_{s}^{+}=-e \cdot\left(\dot{Y}_{b}^{-}-\dot{Y}_{s}^{-}\right)
$$

145 Practically, the problem is reduced to free flight phases (see Eq. 44, with an initial condition for $\dot{Y}_{b}$ given by Eq. 5 . 
In the second approach, the contact phase is governed by the Hertz damped contact model. So the contact force applied on the ball depends on the interpenetration $\delta$ defined as

$$
\begin{array}{cc}
\delta=Y_{b}-Y_{s}-b & \text { if } Y_{b}-Y_{s} \geq b \\
\delta=Y_{b}-Y_{s}+b & \text { if } Y_{b}-Y_{s} \leq-b
\end{array}
$$

In the case of a sphere in contact with a plate, the contact stiffness $K$ considered here is the one given by the Hertz model and defined as

$$
K=\frac{4 \cdot R^{1 / 2}}{3 \cdot \pi \cdot\left(k_{b}+k_{s}\right)}
$$

${ }_{150} \quad$ where the subscripts $b$ and $s$ denote the ball and the structure, respectively, and $k_{i}=\frac{1-\nu_{i}^{2}}{\pi \cdot E_{i}}(i \in\{b, s\})$. The contact force of the Hertz damped model is

$$
F_{\text {contact }}=-K . \delta^{3 / 2}-c . \dot{\delta}
$$

in accordance with the sign convention of Fig. 8 and $\delta$ is defined by Eq. 6 and 7 In Eq. 9 , is the damping coefficient. As it will be explained later, $c$ can be chosen to represent the same energy loss than the one represented by the COR $e$.

This time, the contact duration is not neglected and the equation of motion that governs the motion of the ball during the contact phase is

$$
m_{b} . \ddot{Y}_{b}=-m_{b} . g+F_{\text {friction }}+F_{\text {contact }}
$$

The entire motion of the ball can be simulated using an ODE solver to integrate the equation of motion given by Eq. 4 for the COR model, or Eq. 10 in the case of the Hertz damped contact model.

As the COR model traduces the energy loss by the system during the impact, the Hertz damped model must be able to represent the same dissipation. In the Hertz damped contact model, the dissipation is due to the damping coefficient $c$. Indeed, this contact damping creates an opposing force to the ball displacement that contributes to slow the ball and implies an irreversible kinetic energy loss. In [19], Nagurka et al. provide an approximation that relates the damping coefficient and the COR so that the COR model and a Hertz damped contact model lead to the same dissipation :

$$
c=-\frac{2 \cdot \sqrt{K_{\operatorname{lin} .} \cdot m} \cdot \ln e}{\sqrt{\pi^{2}+(\ln e)^{2}}}
$$

where $K_{\text {lin }}$ denotes the linearised contact stiffness for an average interpenetration $\delta_{a}$ defined as

$$
K_{\text {lin }}=\frac{3}{2} \cdot K \cdot \delta_{a}^{1 / 2}
$$




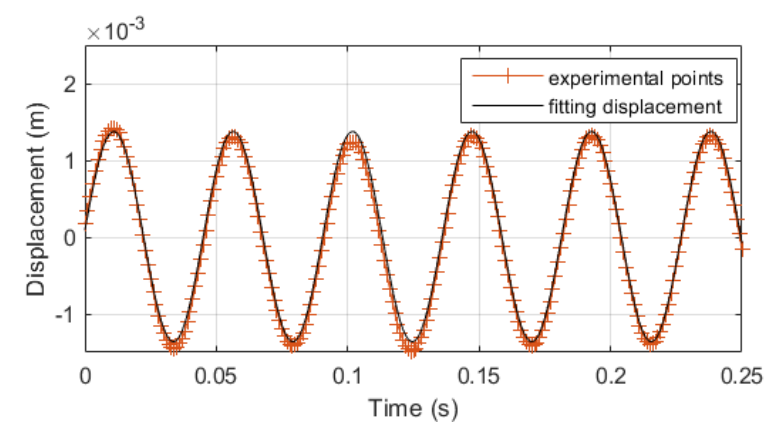

(a) Structure displacement

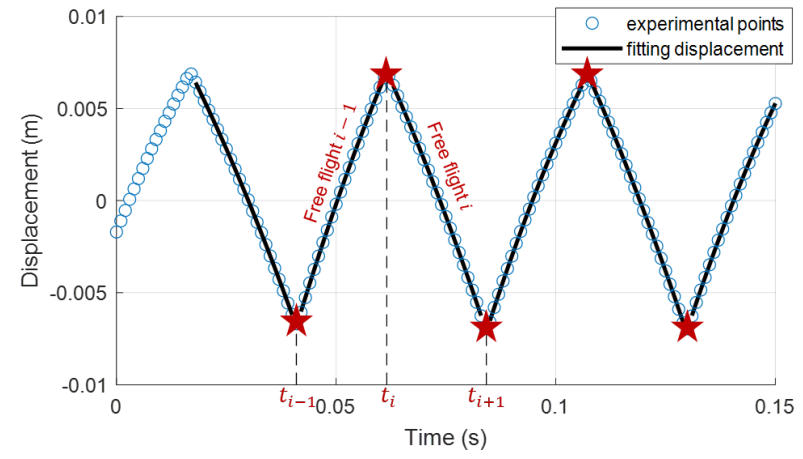

(b) Ball displacement

Figure 9: Fitting displacements for the main structure and the ball

\subsection{Parameter estimation}

In the previous section, discrete-time displacements of the ball and the structure are obtained thanks to the DIC method. In order to obtain velocities, many approaches are possible. In the context of DIC, some authors have used finite differences to estimate velocities and acceleration knowing the displacement field [20, but this method is noise sensitive and leads to inaccuracy in the process of velocity estimation. In order to reduce the uncertainties or to improve the spatial resolution of the results, others have used a method to regularise the displacement, taking in account a set of images instead of using only two pictures corresponding to the reference and deformed configuration [21]. The method used in this paper is to identify the parameters of displacement equations (Eq. 2 and 3 from curve fitting using the experimental displacement points obtained from DIC method. As a result, $A, B, \omega$ and $\varphi$ for the structure, and $a_{i}, b_{i}$ and $c_{i}$ for the ball are obtained minimising the least-square functions :

$$
\begin{gathered}
A, B, \omega, \varphi=\arg \min _{A, B, \omega, \varphi} \sum_{k=1}^{N}\left[Y_{s}\left(t_{k}\right)-Y_{s}^{e x p}\left(t_{k}\right)\right]^{2} \\
a_{i}, b_{i}, c_{i}=\arg \min _{a_{i}, b_{i}, c_{i}} \sum_{k=1}^{N}\left[Y_{b}^{i}\left(t_{k}\right)-Y_{b}^{e x p}\left(t_{k}\right)\right]^{2}
\end{gathered}
$$

where $\mathrm{N}$ is the length of time vector, $Y_{s}^{\exp }$ and $Y_{b}^{\exp }$ are the experimental displacements of the structure and the ball. The fitting displacements are represented in Fig. 9

It is now possible to calculate the velocity of the main structure and ball derivating Eq. 2 and 3 .

Thus, displacements and velocities equations can be used to estimate the kinematics quantities relative to the main structure and ball at every moment, and the whole motion is reconstructed.

With the discrete-time displacements, impact times remain unknown as contact phases are very short and happen between two images. In Fig. 9b, one can see that impacts occur at the intersection of each free flight trajectory (see Fig. 9b), so impact time $t_{i}$ between freeflight $i-1$ and freeflight $i$ can be deduced with Eq. 15

$$
\left(a_{i}-a_{i-1}\right) \cdot t_{i}^{2}+\left(b_{i}-b_{i-1}\right) \cdot t_{i}+c_{i}-c_{i-1}=0
$$

It is now possible to calculate the velocities when the impact occurs to calculate the COR, defined as

$$
e_{i}=\frac{\dot{Y}_{b}^{i}\left(t_{i}\right)-\dot{Y}_{s}\left(t_{i}\right)}{\dot{Y}_{b}^{i-1}\left(t_{i}\right)-\dot{Y}_{s}\left(t_{i}\right)}
$$




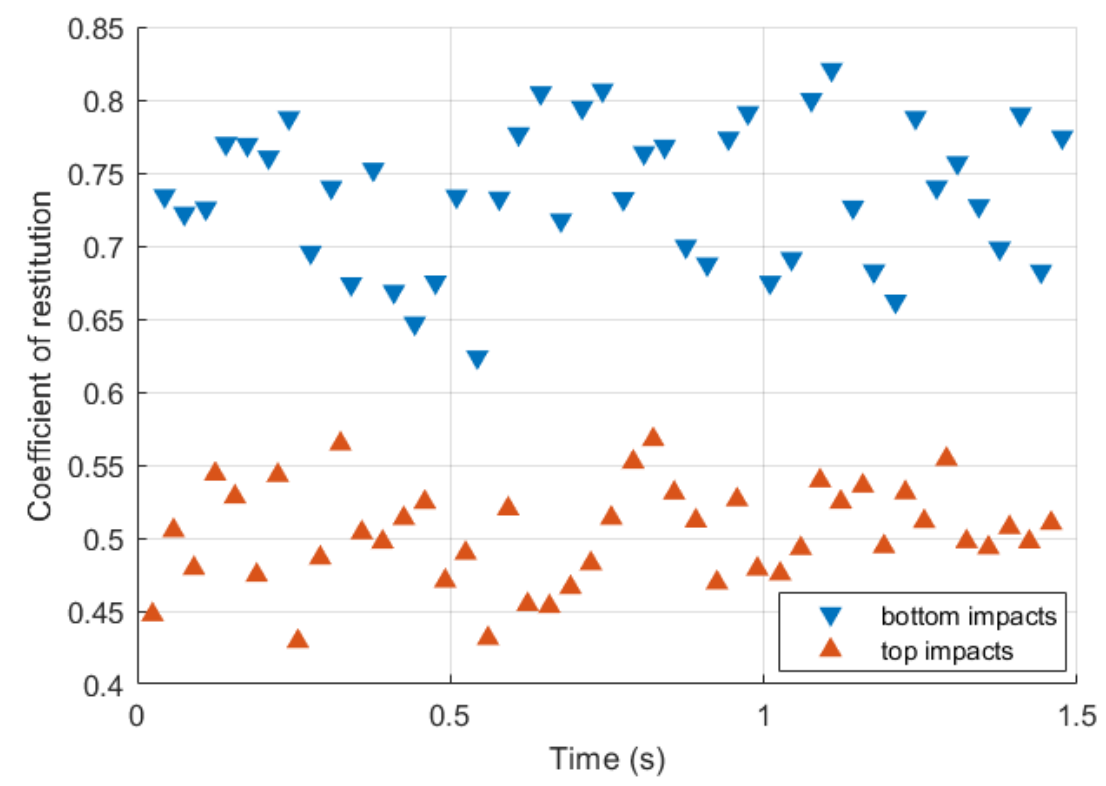

Figure 10: Example of experimental results of the COR (4-th test)

\begin{tabular}{|c|c|c|c|c|}
\hline & $e_{b}$ & $e_{t}$ & $\sigma_{b}$ & $\sigma_{t}$ \\
\hline Test 1 & 0,76 & 0,65 & $3,37.10^{-2}$ & $4,69.10^{-2}$ \\
\hline Test 2 & 0,74 & 0,59 & $4,9.10^{-2}$ & $3,47 \cdot 10^{-2}$ \\
\hline Test 3 & 0,72 & 0,60 & $5,29.10^{-2}$ & $5,06 \cdot 10^{-2}$ \\
\hline Test 4 & 0,73 & 0,50 & $4,89.10^{-2}$ & $3,38 \cdot 10^{-2}$ \\
\hline
\end{tabular}

Table 2: Summary of average COR values and standard deviations (b: bottom. $\mathrm{t}$ : top)

\section{Results of VI characterisation}

In this section, the results of the characterisation of the VI absorber designed for this study are presented and

fall into two parts. The first one is the measure of the COR, and the second one is the measure of the friction forces acting on the ball during its free flight phases.

\subsection{Coefficient Of Restitution}

The COR is calculated for each impact using Eq. 16. An example of the values measured for the fourth test is presented in Fig. 10. Table 2 summarises the mean value of the COR on the lower and upper plate and their standard deviations for each test.

As one can see, the COR is different whether the impact occurs on the upper plate or on the lower plate. This unexpected phenomenon has already been reported in [16] but no special investigation has been made to explain this result. In the present case, as the structure is totally symmetric in terms of materials, assuming that dissipation during impact is only due to contact effects is probably insufficient. Further experiments have been made to try to determine if gravity could influence the COR and provide an explanation of the differences of COR observed between the upper plate and the lower plate. Therefore, the same testing conditions than in test 4 (see Table 1) 


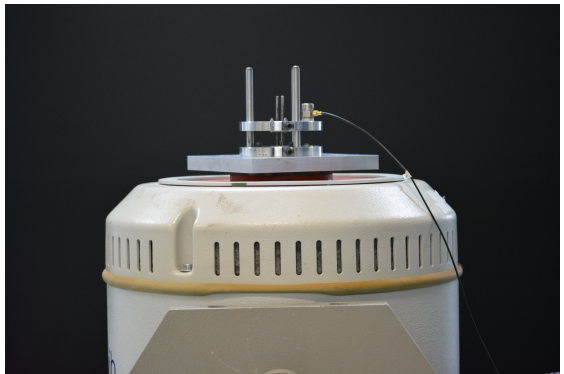

(a) vertical

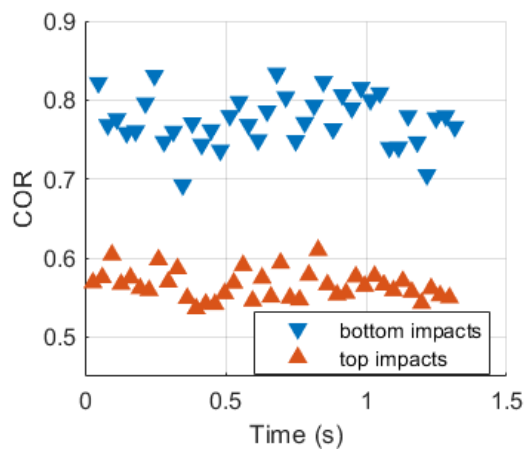

(d) vertical : $e_{b}=0,77 ; e_{t}=0,57$

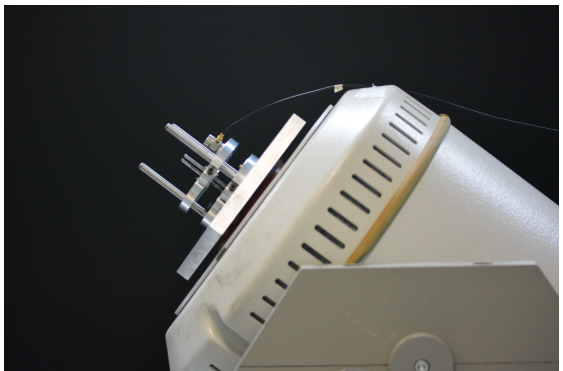

(b) tilted $\left(45^{\circ}\right)$

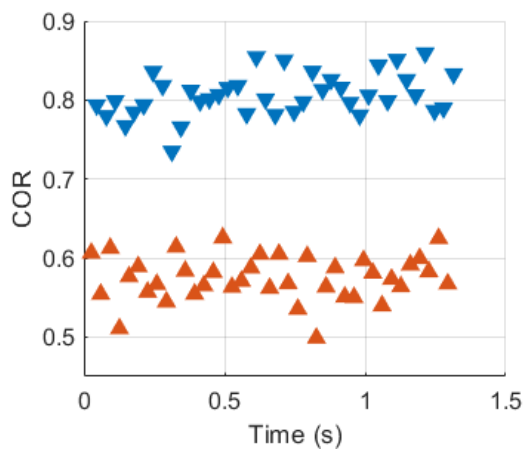

(e) tilted $\left(45^{\circ}\right): e_{b}=0,81 ; e_{t}=0,57$

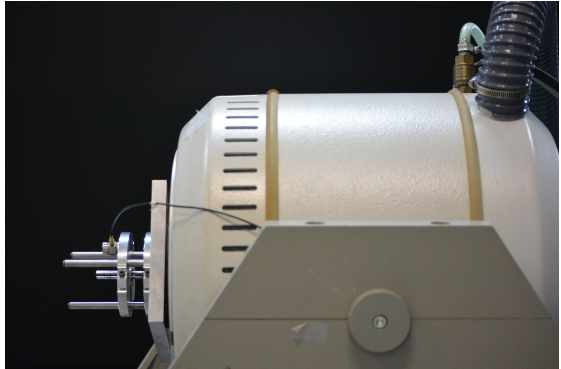

(c) horizontal

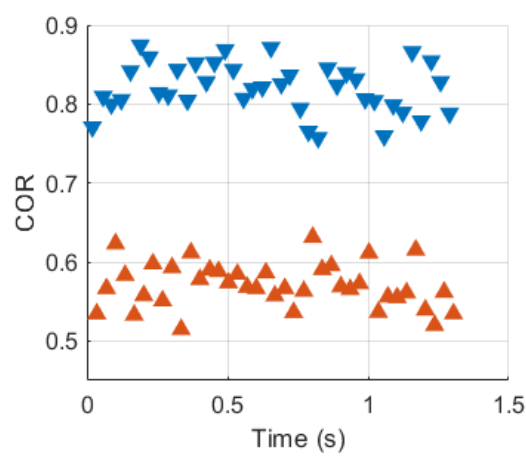

(f) horizontal : $e_{b}=0,82 ; e_{t}=0,57$

Figure 11: Influence of gravity on the coefficient of restitution : pictures of the set-up and COR values

were reproduced for three direction of the set-up, namely vertical, tilted ( 45 degrees from vertical), and horizontal. The results are presented in figure 11 . The average values of the COR for the top and bottom impacts are given in the figure. The COR values are not significantly changed by the direction of the set-up, and the top and bottom values are still different whatever the direction. As a consequence gravity does not seem to be the predominating parameter determining the value of the COR.

Other authors have established a link between the COR and vibrational energy, considering mass-springs elements [22]. Therefore, a certain part of the initial kinetic energy of the ball is lost because converted into vibrational energy of the main structure and leads to a COR smaller than unity. In particular, this phenomenon is well shown in 1 where longitudinal impacts of a ball on rods are studied. The link between the COR and the vibrational energy of the rod after impact is clearly demonstrated. In the field of sport and especially tennis and golf, studies have been made to understand which design parameters influence the COR 22, 24, 25, 26, 27, 28. As presented earlier, two main trends emerge. In the first one, the variation of the COR is studied depending on the materials encountered by the ball or string tension in tennis rackets [27, 24, 26]. In the other approach, the authors try to link the COR with the dynamic properties of the structure that collide. For instance, the authors in [27, 28] have studied the effect of impact location on the COR. In [28, it is shown that the COR is higher when the ball hits the racket close to its vibration node (for the first bending mode). In [25, a link between the natural frequencies of the solids that collide and the COR is established.

These results and the previous work briefly presented here confirm the existence of dynamic phenomena during impact that modify the COR and thus the energy dissipation. In the experiments presented in this paper, various phenomena can occur. As the set-up is an assembly of several parts, its dynamic behaviour in response to an impact 


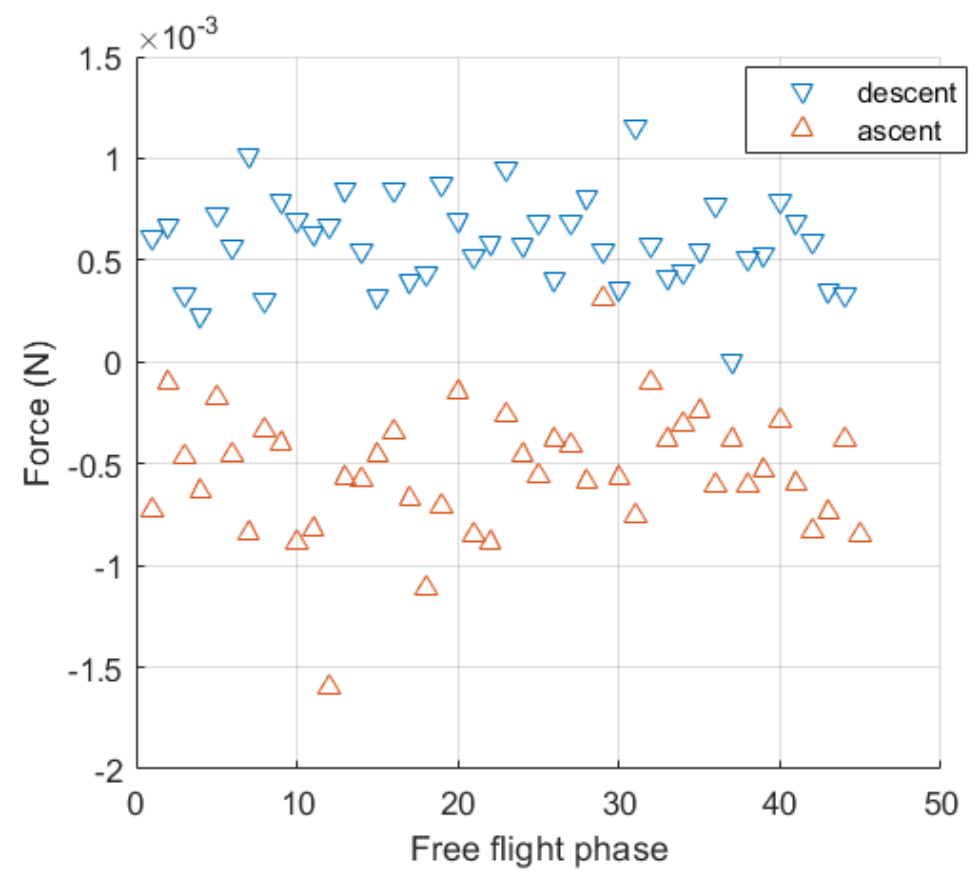

Figure 12: Example of friction forces (4-th test)

is hard to predict, but assuming that the structure is stiffer at its bottom (the side screwed on the shaker), the response of the structure to the impact of the ball might be different when the impact occurs on the lower or on the upper plate, so the COR might be changed. These assumptions should be verified in further experiments and are beyond the objectives of this work.

\subsection{Friction forces}

The identification of the parameters $a_{i}$ provides information on the forces acting on the ball during its free flight phase. Indeed, the dynamic law states that the product of the mass of the ball by its acceleration is equal to the forces acting on it. The acceleration of the ball can be derived from Eq. 3 and is given by $2 . a_{i}$. In the case where no friction forces act on the ball, its acceleration must be equal to the acceleration of gravity $-g$. However, it was found that the acceleration of the ball was slightly different from $-g$, what can be explained by the existence of a friction force $F_{\text {friction }}^{i}$ that acts on the ball during the $i-t h$ free flight phase. Therefore, numerical values were obtained from the difference between the acceleration of the ball and acceleration of gravity :

$$
F_{\text {friction }}^{i}=m_{b} \cdot\left(2 \cdot a_{i}+g\right)
$$

These forces are represented in Fig. 12. It is relevant to notice that these forces are effectively opposed to the motion of the ball, except for the $29^{t h}$ ascent free flight where the value is not consistent.

\subsection{Simulation of the VI model}

The results presented in the two previous sections allow to characterise the contact phases with the COR for each impact, and the free flight phases with the friction forces for each free flight. These results, for each contact phase and free flight phase, can be used in the models presented in section 2 and they can be simulated. In Fig. 13 , 


\begin{tabular}{|c|c|c|c|c|}
\hline & Test 1 & Test 2 & Test 3 & Test 4 \\
\hline COR model & $2,78 \%$ & $2,51 \%$ & $2,44 \%$ & $3,5 \%$ \\
\hline Hertz damped model & $3,15 \%$ & $3,34 \%$ & $3,43 \%$ & $3,71 \%$ \\
\hline
\end{tabular}

Table 3: Error between experience and simulation for the COR model and Hertz model

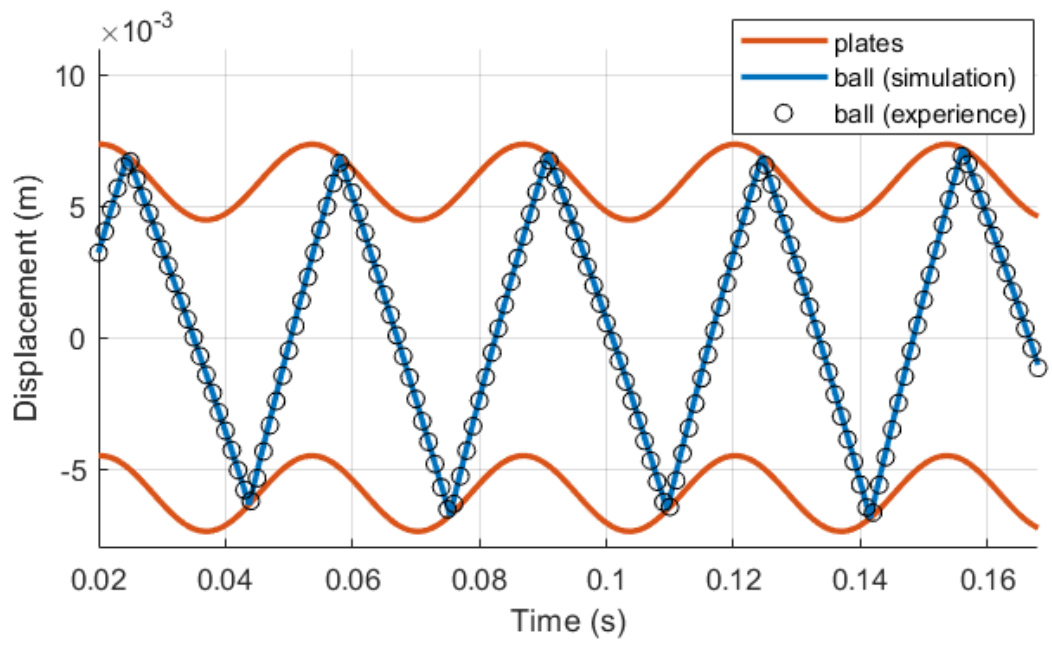

Figure 13: Comparison of experimental and simulated displacement using the COR model

one can see that using the experimental values of the COR and of the friction forces leads to the same behaviour of the VI absorber in simulation than in the experience. The same result is found for the Hertz damped model. The deviation $D$ between experimental and simulation displacements for the ball is quantified using Eq. 18 :

$$
D=\sqrt{\frac{\int_{t_{i}}^{t_{f}}\left(Y_{b}^{\text {simu }}-Y_{b}^{\text {expe }}\right)^{2} \cdot d t}{\int_{t_{i}}^{t_{f}}\left(Y_{b}^{\text {expe }}\right)^{2} \cdot d t}}
$$

where $t_{i}$ and $t_{f}$ denote initial instant and final instant of the experience, respectively. The deviations calculated for the different tests and for both models are presented in Table 3 . As a consequence, it can be concluded that the motion reconstruction method presented in this paper is able to perform accurate measurements of the position of both the ball and the main structure in the configuration chosen for the tests, and that the models proposed before are relevant in these cases.

\section{Discussion : statistical analysis of experimental data and uncertainties}

As presented in section 4 the COR values are scattered around an average value (depending on the plate where the impact occurs). The COR values are analysed from a statistical point of view. To determine if the values follow a special distribution, histograms are plotted in Fig. 15. $\mu$ and $\sigma$ denote the average value and the standard deviation respectively. As normal distribution seems to fit the experimental data, quantile-quantile diagrams are plotted in Fig. 16. High correlation coefficients suggest that the COR values follow a normal distribution. Similar results were found for the other tests. Average values for the 4 tests with standard deviation error bars $( \pm \sigma)$ are 


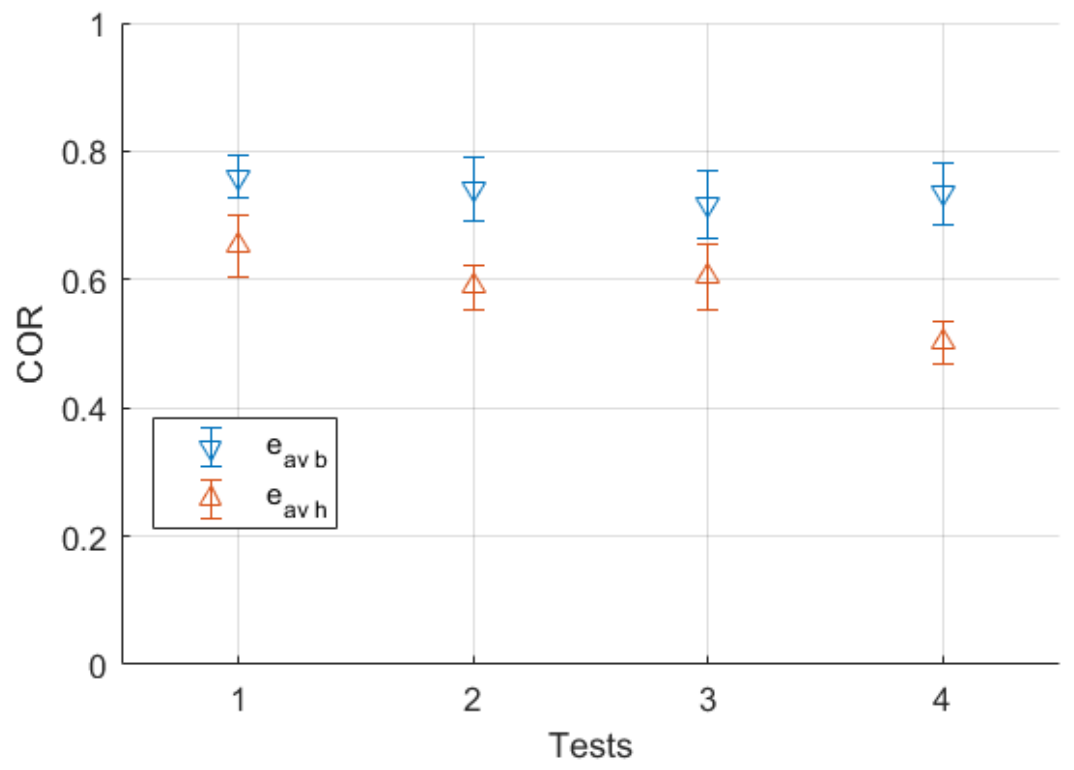

Figure 14: Summary of average COR values with error bars $( \pm \sigma)$

presented in Fig. 14. Comparatively to these results, the COR values presented in [16] seem to be scattered in a similar manner, and the standard deviations given in 14 are consistent with the one presented in this paper.

Further work should be done to explain the origin of the scattering of the measurements. In [29], the authors have made a movie of a motionless specimen (a disk in compression between Hopkinson bars) to quantify the uncertainty on the gray level between pictures because of noised pictures. The same methodology is applied here to determine the noise displacement involved by noise on the pictures. Such a displacement is represented in Fig. 17. One can see that the displacement can be divided into a high frequency content and a low frequency content. The first part can be attributed to the noise on pictures, that leads to accuracy loss in the correlation process. The second part is very likely to be due to unwanted motion of the camera while recording the pictures. As the total displacement is approximately 100 times lower than the motion amplitude of the ball (and 50 times for the structure), it is reasonably low to assume that its effects on the displacement reconstruction and COR estimation are negligible. Moreover, here is the main advantage of the reconstruction method proposed in this paper. Using a model associated to a curve fitting process, the noise existing on the measures has a negligible influence on the displacement and velocity estimation, whereas using classical finite differences to determine the velocity is wellknown to be very noise sensitive, what leads to a bad estimation of the velocity. Other sources of uncertainty in the COR estimation can be identified, such as the non horizontality of the camera or the rotation of the ball. As an angle of the camera to the horizontal does not modify the measure of the COR because the relative velocities of Eq. 5 in each side of the equation are multiplied by the same coefficient (depending on the angle), this cannot be responsible of the scattering of the COR values. Concerning the rotation of the ball, it is demonstrated in [14] that the six degrees of freedom of the ball must be considered to determine repeatable values of the COR (i.e with a small standard deviation) for a bouncing ball on a plane. In the present case, only the vertical translation is possible, but rotations around the three axis are a priori possible, but not considered here to calculate the COR. 


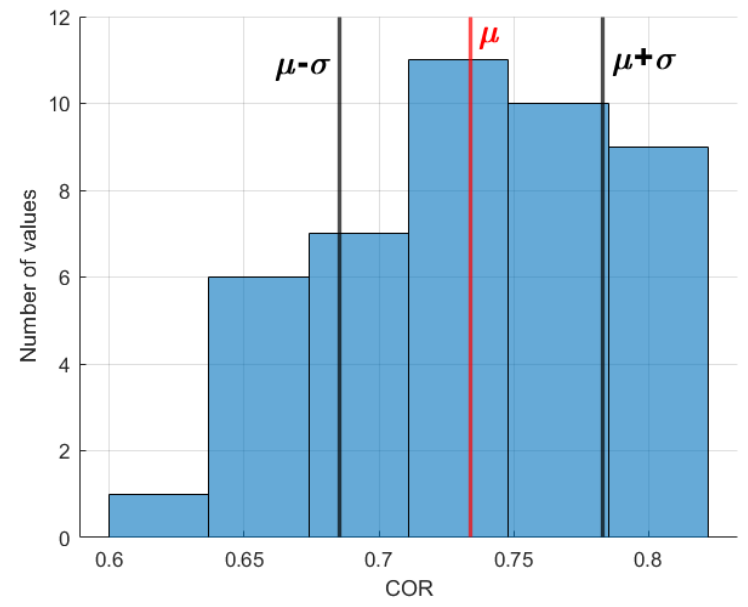

(a) Lower plate

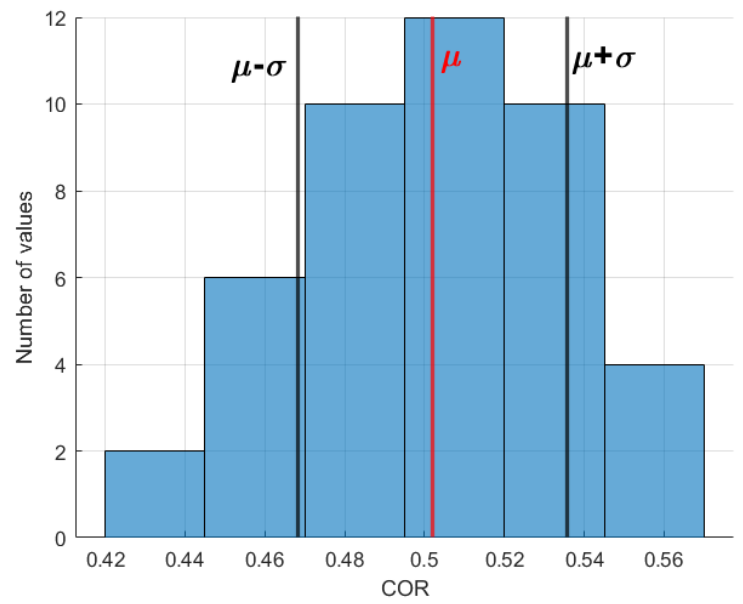

(b) Upper plate

Figure 15: Histogram of COR values (4-th test)

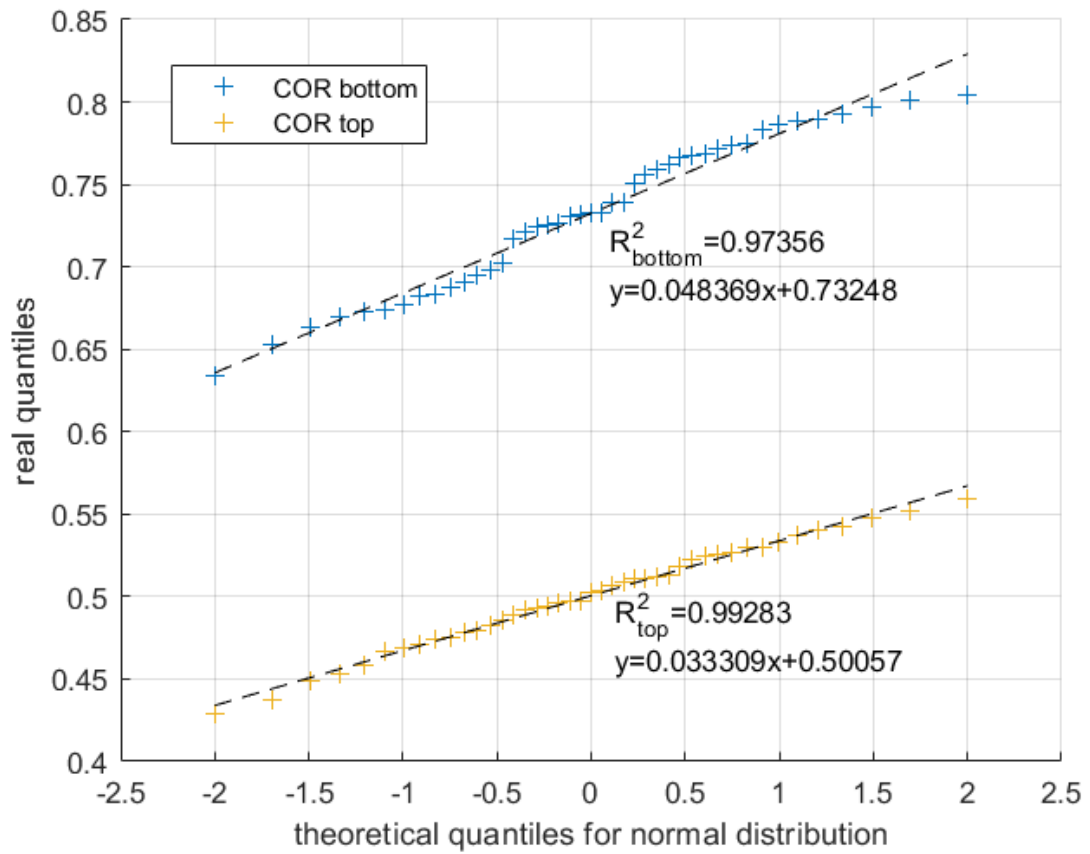

Figure 16: Quantile-quantile diagram for normal distribution (4-th test) 


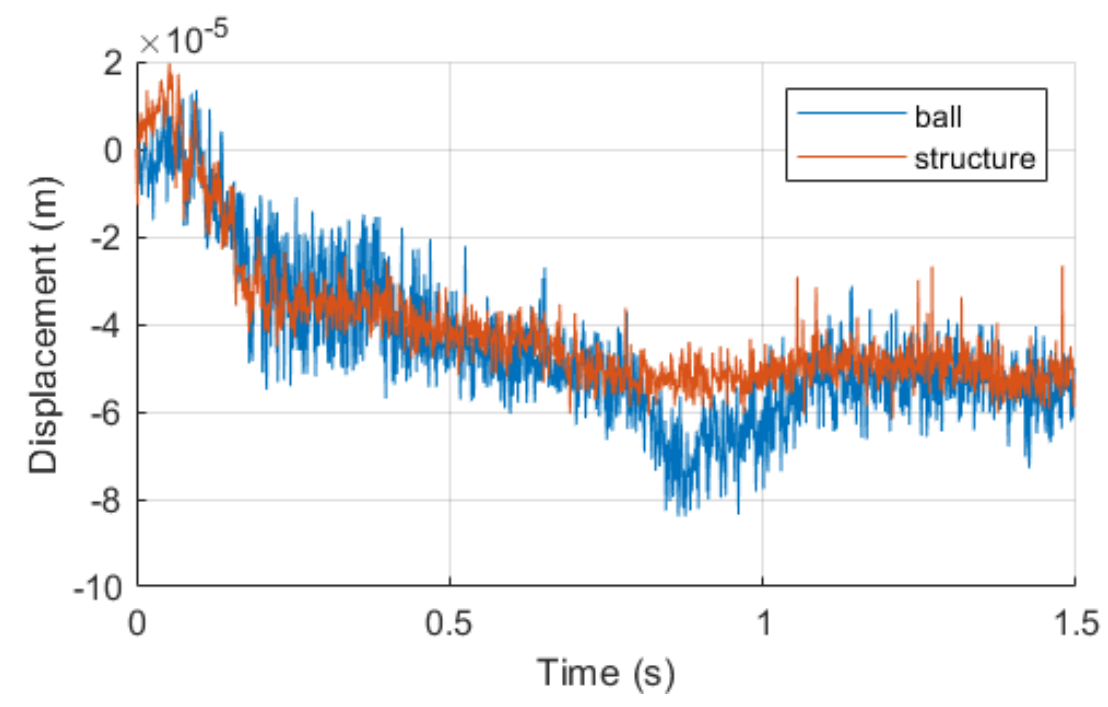

Figure 17: Noise displacements measured during motionless test

Moreover, such rotations of the ball may be induced by the friction forces identified previously, in the case where they correspond to friction on the guiding rods. Nevertheless, the ceramic ball is too smooth and shiny to visualise its rotation.

No special work has been done to find the origin of the friction forces identified in this paper. But two assumptions can be made. These forces can be due to friction on the guiding rods when the ball is in contact with them. Another source of friction can be the aerodynamic drag of the ball. Further work could be done to try to estimate the proportion of each category of force, and to understand how to design a set-up to control the effect of their intensity. Such results could be linked with [3] in which the effect of friction on the absorber efficiency is analysed.

\section{Conclusions}

In this study, an experimental set-up has been designed to take advantage of Digital Image Correlation and full-field measurement using a high-speed camera in order to reconstruct the motions of the main-structure and the free oscillating mass of a vibro-impact absorber. This method overcomes the difficulties encountered to measure the displacement of the oscillating mass with conventional sensors. Once the discrete-time displacements are obtained from Digital Image Correlation, continuous displacements are derived identifying models parameters using curve fitting on the experimental points. Velocities can be deduced with the analytical derivative of displacements without amplifying the noise, and the coefficient of restitution can be calculated. With this method, impact instants can be accurately determined even if it occurs between two images recorded by the camera. Moreover, friction forces are evaluated during the free flight phase of the oscillating mass. The method turns out to be accurate in the different configurations tested, as the agreement with the simulated models is very good. Experimental characterisation of a vibro-impact absorber can now be realised, and allows to determine the coefficient of restitution and friction forces that can be used in a model. The dynamic response of the structure highlights that a high-frequency energy transfer exists because of vibro-impact phenomenon, which could be one of the source of dissipation during impact, leading to a coefficient of restitution smaller than unity. It was demonstrated that gravity has no significant influence on 
the coefficient of restitution, and further work must be done in order to explain more precisely the origin of the

differences when measuring the coefficient of restitution on the upper plate or on the lower plate.

\section{Acknowledgment}

This work has been supported by the EUR EIPHI Project (contract ANR-17-EURE-0002) and Bourgogne Franche-Comté Region.

\section{Declaration of interest}

The authors declare that they have no known competing financial interests or personal relationships that could have appeared to influence the work reported in this paper.

\section{Appendix A. Appendix : Features of the experimental set-up}

The mass of the ball is $1,82.10^{-4} \mathrm{~kg}$, and the mass of the entire structure of the VI absorber is $6,63.10^{-1} \mathrm{~kg}$. The materials used for each part are summurised in Table A.4. The main dimensions are given in Fig. A.18. During all the tests presented in this paper, the distance between the upper disk and the lower disk was $16.6 \mathrm{~mm}$.

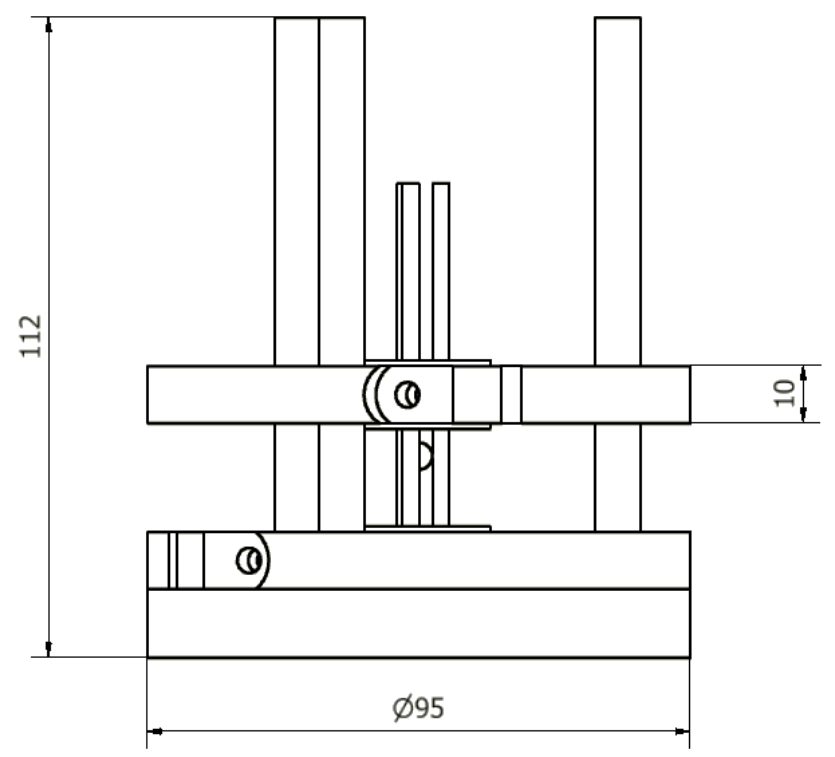

Figure A.18: Main dimensions (in $\mathrm{mm}$ ) of the experimental set-up 


\begin{tabular}{|c|c|}
\hline & material \\
\hline ball & ceramic \\
\hline plates & aluminium \\
\hline guiding and holding rods & steel \\
\hline disks & aluminium \\
\hline
\end{tabular}

Table A.4: Materials of the experimental set-up of the VI damper 


\section{References}

[ [1] R. Seifried, W. Schiehlen, P. Eberhard, Numerical and experimental evaluation of the coefficient of restitution

口 for repeated impacts, International Journal of Impact Engineering 32 (1-4) (2005) 508-524. doi:10.1016/j. ijimpeng.2005.01.001.

URL https://linkinghub.elsevier .com/retrieve/pii/S0734743X05000114

口 [2] R. Boettcher, S. Eichmann, P. Mueller, Influence of viscous damping and elastic waves on energy dissipation during impacts, Chemical Engineering Science 199 (2019) 571-587. doi:10.1016/j.ces.2019.01.036. URL https://linkinghub.elsevier.com/retrieve/pii/S0009250919301186

¿ [3] T. Theurich, J. Gross, M. Krack, Effects of modal energy scattering and friction on the resonance mitigation with an impact absorber, Journal of Sound and Vibration 442 (2019) 71-89. doi:10.1016/j.jsv.2018.10.055. URL https://linkinghub.elsevier .com/retrieve/pii/S0022460X18307351

[4] P. L. Reu, T. J. Miller, The application of high-speed digital image correlation, The Journal of Strain Analysis for Engineering Design 43 (8) (2008) 673-688. doi:10.1243/03093247JSA414

URL http://journals . sagepub.com/doi/10.1243/03093247JSA414

[5] V. Tiwari, M. A. Sutton, S. McNeill, S. Xu, X. Deng, W. L. Fourney, D. Bretall, Application of 3D image correlation for full-field transient plate deformation measurements during blast loading, International Journal of Impact Engineering 36 (6) (2009) 862-874. doi:10.1016/j.ijimpeng.2008.09.010.

URL https://linkinghub.elsevier.com/retrieve/pii/S0734743X08002376

q [6] G. Besnard, F. Hild, J.-M. Lagrange, P. Martinuzzi, S. Roux, Analysis of necking in high speed experiments by 305 stereocorrelation, International Journal of Impact Engineering 49 (2012) 179-191. doi:10.1016/j.ijimpeng. 2012.03.005.

URL https://linkinghub.elsevier.com/retrieve/pii/S0734743X1200070X

[ [7] V. Tarigopula, O. Hopperstad, M. Langseth, A. Clausen, F. Hild, A study of localisation in dual-phase highstrength steels under dynamic loading using digital image correlation and FE analysis, International Journal of Solids and Structures 45 (2) (2008) 601-619. doi:10.1016/j.ijsolstr.2007.08.021. URL https://linkinghub.elsevier.com/retrieve/pii/S0020768307003411

[ [8] D. Saletti, S. Pattofatto, H. Zhao, Measurement of phase transformation properties under moderate impact tensile loading in a NiTi alloy, Mechanics of Materials 65 (2013) 1-11. doi:10.1016/j .mechmat.2013.05.017. URL https://linkinghub.elsevier.com/retrieve/pii/S0167663613001099

315 _ [9] J. Horabik, M. Beczek, R. Mazur, P. Parafiniuk, M. Ryżak, M. Molenda, Determination of the restitution coefficient of seeds and coefficients of visco-elastic Hertz contact models for DEM simulations, Biosystems Engineering 161 (2017) 106-119. doi:10.1016/j.biosystemseng.2017.06.009. URL https://linkinghub.elsevier.com/retrieve/pii/S1537511017303604 
[10] C. Wong, M. Daniel, J. Rongong, Energy dissipation prediction of particle dampers, Journal of Sound and Vibration 319 (1-2) (2009) 91-118. doi:10.1016/j.jsv.2008.06.027.

URL https://linkinghub.elsevier.com/retrieve/pii/S0022460X08005646

[11] H. Dong, M. Moys, Experimental study of oblique impacts with initial spin, Powder Technology 161 (1) (2006) 22-31. doi:10.1016/j.powtec.2005.05.046.

URL https://linkinghub.elsevier.com/retrieve/pii/S0032591005002688

12] L. Wang, B. Wu, Z. Wu, R. Li, X. Feng, Experimental determination of the coefficient of restitution of particle-

particle collision for frozen maize grains, Powder Technology 338 (2018) 263-273. doi:10.1016/j.powtec. 2018.07 .005 .

URL https://linkinghub.elsevier.com/retrieve/pii/S0032591018304947

[13] D. Hastie, Experimental measurement of the coefficient of restitution of irregular shaped particles impacting on horizontal surfaces, Chemical Engineering Science 101 (2013) 828-836. doi:10.1016/j.ces.2013.07.010. URL https://linkinghub.elsevier.com/retrieve/pii/S0009250913005022

[14] J. E. Higham, P. Shepley, M. Shahnam, Measuring the coefficient of restitution for all six degrees of freedom, Granular Matter 21 (2) (2019) 15. doi:10.1007/s10035-019-0871-0.

URL http://link.springer.com/10.1007/s10035-019-0871-0

[15] E. Rigaud, J. Perret-Liaudet, Investigation of gear rattle noise including visualization of vibro-impact regimes, Journal of Sound and Vibration 467 (2020) 115026. doi:10.1016/j.jsv.2019.115026.

URL http://www.sciencedirect.com/science/article/pii/S0022460X19305899

[16] T. Li, S. Seguy, C.-H. Lamarque, A. Berlioz, Experiment-Based Motion Reconstruction and Restitution Coefficient Estimation of a Vibro-Impact System, Journal of Vibration and Acoustics 141 (2) (2019) 021003. doi:10.1115/1.4041367 URL https://asmedigitalcollection.asme.org/vibrationacoustics/article/doi/10.1115/1. 4041367/475092/ExperimentBased-Motion-Reconstruction-and

[17] G. Besnard, Caractérisation et quantification de surfaces par stéréocorrélation pour des essais mécaniques du quasi statique à la dynamique ultra-rapide 289.

[18] L. Serdukova, R. Kuske, D. Yurchenko, Stability and bifurcation analysis of the period-T motion of a vibroimpact energy harvester, Nonlinear Dynamics 98 (3) (2019) 1807-1819. doi:10.1007/s11071-019-05289-8.

URL http://link.springer.com/10.1007/s11071-019-05289-8

[19] M. Nagurka, Shuguang Huang, A mass-spring-damper model of a bouncing ball in: Proceedings of the 2004

4. American Control Conference, IEEE, Boston, MA, USA, 2004, pp. 499-504 vol.1. doi:10.23919/ACC.2004. 350 1383652 .

URL https://ieeexplore.iee. org/document/1383652/ 
[20] B. Koohbor, A. Kidane, M. A. Sutton, X. Zhao, S. Mallon, Analysis of dynamic bending test using ultra high speed DIC and the virtual fields method, International Journal of Impact Engineering 110 (2017) 299-310. doi:10.1016/j.ijimpeng.2016.12.021

URL https://linkinghub.elsevier.com/retrieve/pii/S0734743X16306340

[21] G. Besnard, H. Leclerc, F. Hild, S. Roux, N. Swiergiel, Analysis of image series through global digital image

a correlation, The Journal of Strain Analysis for Engineering Design 47 (4) (2012) 214-228. doi:10.1177/ 0309324712441435

URL http://journals . sagepub.com/doi/10.1177/0309324712441435

[22] H. Ruan, T. Yu, The unexpectedly small coefficient of restitution of a two-degree-of-freedom mass-spring system

․ and its implications, International Journal of Impact Engineering 88 (2016) 1-11. doi:10.1016/j.ijimpeng. 2015.09 .005 .

URL https://linkinghub.elsevier.com/retrieve/pii/S0734743X15001955

[23] P. Ray, G. Chahine, P. Smith, R. Kovacevic, Optimal Design of a Golf Club using Functionally Graded Porosity (2011).

URL https://www.researchgate.net/profile/Radovan_Kovacevic/publication/268433480_Optimal_ Design_of_a_Golf_Club_using_Functionally_Graded_Porosity/links/54be5e430cf218da9391e7ca/ Optimal-Design-of-a-Golf-Club-using-Functionally-Graded-Porosity·pdf

[24] A. Vallatta, F. Casolo, M. Caffi, On The Coefficient Of Restitution Of Tennis Rackets (1993). URL https://ojs.ub.uni-konstanz.de/cpa/article/view/1709

[25] Numerical Examinations of Relation between Restitution Characteristic and Eigen Frequencies $\mid$ Elsevier Enhanced Reader, library Catalog: reader.elsevier.com. doi:10.1016/j.proeng.2011.04.543.

URL https://reader.elsevier.com/reader/sd/pii/S1877705811007314?token= A5BDC9468094346629E958AFC410C963CFE707FE6841173B5BE0F2FB4781CD2AEACAB3F97AD1BC446B35158AE9533E2E

375 [26] C. B. A. D. H. L. e. N. L. Manin, R. Rinaldi, Impact normal d'une sphere creuse en plastique sur une couche plane en polymere: application au tennis de table, Le Mans, 2016.

URL http://www . conforg.fr/cfa2016/cdrom/data/articles/000512.pdf

[27] L. Li, S. H. Yang, C.-S. Hwang, Y. S. Kim, Effects of string tension and impact location on tennis playing, Journal of Mechanical Science and Technology 23 (11) (2009) 2990-2997. doi:10.1007/s12206-009-0903-5. URL http://link.springer.com/10.1007/s12206-009-0903-5

[28] Y. Kawazoe, Y. Kanda, Analysis of Impact Phenomena in a Tennis Ball-Raket System, JSME International Journal (1997).

URL https://www .jstage .jst.go.jp/article/jsmec1993/40/1/40_1_9/_pdf/-char/ja

[29] F. Hild, A. Bouterf, S. Roux, Measurement of kinematic fields via DIC for impact engineering applications, International Journal of Impact Engineering 130 (2019) 163-171. doi:10.1016/j.ijimpeng.2019.04.007. URL https://linkinghub.elsevier.com/retrieve/pii/S0734743X1831087X 